\title{
DIFFERENTIAL INVARIANT THEORY OF ALTERNATING TENSORS*
}

CLYDE M. CRAMLET

1. Introduction. In a former paper $\dagger$ a general method was developed for obtaining a complete system of tensors for a general $n$-ary $q$-ic differential form. The quantities $\Lambda_{r_{1}} \ldots r_{q^{s}}$ of that article are proportional to the quantities $a_{r_{1} \ldots r_{q}}$ of this paper which do not contain the second derivatives when the fundamental tensor is alternating. Thus that method for establishing convariant differentiation with respect to a covariant $q$-ic form fails when the form is alternating. This exceptional case will be treated here.

Under an analytic transformation of coordinates

$$
x^{i}=x^{i}(\bar{x}), \quad i=1, \cdots, n, \quad\left|\frac{\partial x^{r}}{\partial \bar{x}^{s}}\right| \neq 0,
$$

the alternating covariant tensor $a_{r_{1}} \cdots r_{q}$ transforms by the equations

$$
\bar{a}_{r_{1}} \cdots r_{q}=a_{\rho_{1}} \cdots \rho_{q} p_{r_{1}}^{\rho_{1}} \cdots p_{r_{q}}^{\rho_{q}}, \quad p_{s}^{r}=\frac{\partial x^{r}}{\partial \bar{x}^{s}} .
$$

The property of being alternating is invariant.

We propose to find conditions under which these equations with preassigned $\bar{a}_{r_{1}} \cdots r_{q}$ and $a_{r_{1}} \cdots r_{q}$ admit solutions $p_{s}{ }^{r},\left|p_{s}{ }^{r}\right| \neq 0$,

$$
p_{s t}^{r}=\underset{t_{s}}{r}, \quad p_{s t}^{r} \equiv \frac{\partial p_{s}^{r}}{\partial \bar{x}^{t}},
$$

and for which the differential equations

$$
\frac{\partial x^{r}}{\partial \bar{x}^{s}}=p_{s}^{r}
$$

are integrable and yield solutions (1) determining a transformation of coordinates.

The statement of the conditions under which such systems admit solutions is contained in a note by the writer which precedes the

* Presented to the Society, December 27, 1934.

$\dagger$ The invariants of an n-ary $q$-ic differential form, Annals of Mathematics, (2), vol. 31 (1930), pp. 134-150. 
present paper in this number of this Bulletin.* The method used here is a direct application of this theory, the $\bar{x}$ 's and $x$ 's here corresponding to the $x$ 's and $z$ 's of that paper, and equations (2) here corresponding to the system $S$ of I.C. Here we require the additional restrictions $\left|p_{s}{ }^{r}\right| \neq 0$.

2. The derived tensors. To proceed to the derivation of the sets of equations in the chain, $\dagger$ we differentiate the first set (2), obtaining,

$$
\begin{aligned}
\frac{\partial \bar{a}_{r_{1}} \cdots r_{q}}{\partial \bar{x}^{s}}= & a_{\rho_{1} \cdots \rho_{q}}\left[p_{r_{1} s}^{\rho_{1}} \cdots p_{r_{q}}^{\rho_{q}}+\cdots+p_{r_{1}}^{\rho_{1}} \cdots p_{r_{i} s}^{\rho_{i}} \cdots p_{r_{q}}^{\rho_{q}}+\cdots\right] \\
& +\frac{\partial a_{\rho_{1}} \cdots \rho^{q}}{\partial x^{\sigma}} p_{r_{1}}^{\rho_{1}} \cdots p_{r_{q}}^{\rho_{q}} p_{s}^{\sigma},
\end{aligned}
$$

$$
\begin{aligned}
& \frac{\partial \bar{a}_{s r_{1}} \cdots r_{q}}{\partial \bar{x}^{r_{1}}}=a_{\rho_{1}} \cdots \rho_{q}\left[p_{s r_{1}}^{\rho_{1}} \cdots p_{r_{q}}^{\rho_{q}}+\cdots+p_{s}^{\rho_{1}} \cdots p_{r_{i} r_{1}}^{\rho_{i}} \cdots p_{r_{q}}^{\rho_{q}}+\cdots\right] \\
& +\frac{\partial a_{\sigma \rho_{2}} \cdots \rho_{q}}{\partial x^{\rho_{1}}} p_{r_{1}}^{\rho_{1}} \cdots p_{r_{q}}^{\rho_{q}} \dot{p}_{s}^{\sigma} \\
& \frac{\partial \bar{a}_{r_{1}} \cdots s r_{r_{q}}}{\partial \bar{x}^{r_{m}}}=a_{\rho_{1}} \cdots \rho_{q}\left[p_{r_{1} r_{m}}^{\rho_{1}} \cdots p_{s}^{\rho_{m}} \cdots p_{r_{q}}^{\rho_{q}}+\cdots\right. \\
& \left.+p_{r_{1}}^{\rho_{1}} \cdots p_{s}^{\rho_{m}} \cdots p_{r_{k} r_{m}}^{\rho_{k}} \cdots p_{r_{q}}^{\rho_{q}}+\cdots\right]+\frac{\partial a_{\rho_{1}} \cdots \sigma \cdots \rho q}{\partial \bar{x}^{\rho_{m}}} p_{r_{1}}^{\rho_{1}} \cdots p_{s}^{\sigma}
\end{aligned}
$$

This last equation is written for $k>m$. A similar term appears for $k<m$. in

Consider any term involving $p_{r_{i} \text { s }}^{\alpha}$. It appears in the first equation

$$
a_{\rho_{1}} \cdots \rho_{q} p_{r_{1}}^{\rho_{1}} \cdots p_{r_{i} s}^{\rho_{i}} \cdots p_{r_{q}}^{\rho_{q}}, \quad i=1, \cdots, n .
$$

This term appears also in the $(i+1)$ th equation as, $a_{\rho_{1}} \ldots \rho_{q} p_{r_{1}}^{\rho_{1}} \cdots p_{s r_{i}}^{\rho_{i}}$ $\cdots p_{q_{q^{*}}}^{\rho_{q}}$ All such terms will cancel if the sum of all equations after the first is subtracted from the first.

Now consider any terms involving $p_{r_{k} r_{m}}^{\alpha}, r_{k} \neq r_{m} \neq s$. There will be two such terms, one appearing in the $(m+1)$ st equation and another in the $(k+1)$ st. It will be convenient to think of $m<k$ but this is not a restriction. The terms referred to are

* Note on integrability conditions of implicit differential equations, this Bulletin, vol. 44 (1938), pp. 107-109. This paper will be referred to as I.C.

$\dagger$ Equation (1), I.C. 
and

$$
a_{\rho_{1}} \cdots \rho_{q} p_{r_{1}}^{\rho_{1}} \cdots p_{s}^{\rho_{m}} \cdots p_{r_{k} r_{m}}^{\rho_{k}} \cdots p_{r_{q}}^{\rho_{q}}
$$

$$
a_{\rho_{1}} \cdots \rho_{q} p_{r_{1}}^{\rho_{1}} \cdots p_{r_{m} r_{k}}^{\rho_{m}} \cdots p_{s}^{\rho_{k}} \cdots p_{r_{q}}^{\rho_{q}} \text {. }
$$

By an interchange of summation indices $\rho_{m}$ and $\rho_{k}$ it appears from the alternating character of $a_{r_{1}} \ldots r_{q}$ that these terms differ only in sign, hence cancel in the sum of all equations after the first. Thus

$$
\begin{aligned}
\bar{a}_{r_{1}} \cdots r_{q^{s}} & =a_{\rho_{1} \cdots \rho_{q} \sigma} p_{r_{1}}^{\rho_{1}} \cdots p_{r_{q}}^{\rho_{q}} p_{s}^{\sigma}, \\
a_{r_{1}} \cdots r_{q^{s}} & \equiv \frac{\partial a_{r_{1}} \cdots r_{q}}{\partial x^{s}}-\frac{\partial a_{s r_{2}} \cdots r_{q}}{\partial x^{r_{1}}}-\cdots-\frac{\partial a_{r_{1}} \cdots r_{q-1_{1}}}{\partial x^{r_{q}}} .
\end{aligned}
$$

These equations may be written

$$
a_{r_{1} \cdots r_{q^{s}}}=\frac{\partial a_{r_{1} \cdots r_{q}}}{\partial x^{s}} \pm \frac{\partial a_{r_{2} \cdots r_{q^{s}}}}{\partial x^{r_{1}}}+\frac{\partial a_{r_{3} \cdots s r_{1}}}{\partial x^{r_{2}}} \pm \cdots
$$

where all signs are positive if $q$ is even, and alternately positive and negative if $q$ is odd, and each term is obtained from the preceding term by a cyclic advance of the indices.

This is the well known* alternating tensor which will be called the first derived tensor. It is also well known that when the tensor appearing in the right member of (8) is a derived tensor the result vanishes identically, or the second derived tensor has the value

$$
a_{r_{1}} \cdots r_{g^{s t}} \equiv 0 \text {. }
$$

3. A complete system of eliminants. Equations (6) are eliminants of (5), consequences of (3). There are no other eliminants, when we assume that (2) and (6) possess simultaneous solutions $p_{s}{ }^{r}$. For (6) may be used to replace the first set of (5). The remaining $q$ sets contain given derivatives $p_{s r_{1}}^{\rho}$ in but a single set, and the $p_{r_{1}{ }^{s}}^{\rho}$ do not appear. Hence no eliminants due to (3) exist. No eliminants independent of (3) are obtainable, for these equations are consistent from the hypothesis that (2) are consistent. Equations (6) must be similarly treated. The resulting equations are like (5) with $q$ replaced by $q+1$ and will be referred to as $\left(5^{\prime}\right)$. Again the first set may be discarded by the use of (9) and the only equations containing the derivatives $p_{s r}^{\rho}$ under discussion above will appear in a single set. The algebraic eliminants obtained by eliminating the $p_{s r_{1}}^{r}$ from (5) and $\left(5^{\prime}\right)$ will be satisfied because of the hypothesis of consistency of (2) and (6) and no eliminants can result from the combined sets (5) and

* Murnaghan, Vectors and Tensors, p. 35. 
$\left(5^{\prime}\right)$ because of (3), for we have shown that, if we consider any derivatives $p_{s t}^{r}$, the independent equations of (5) and $\left(5^{\prime}\right)$ may be so chosen that only $p_{s t}^{r}$ and not $p_{t s}^{r}$ appear in them.

4. The complete chain. The chain of I.C. (1) may now be constructed. The set $F^{1}$ will include the sets (2) and (6):

$$
\begin{aligned}
F^{1}: \quad \bar{a}_{r_{1}} \cdots r_{q} & =a_{\rho_{1}} \cdots \rho_{q} p_{r_{1}}^{\rho_{1}} \cdots p_{r_{q}}^{\rho_{q}}, \\
\bar{a}_{r_{1}} \cdots r_{q^{s}} & =a_{\rho_{1}} \cdots \rho_{q} p_{r_{1}}^{\rho_{1}} \cdots p_{r_{q}}^{\rho_{q} p_{s}^{\sigma}} .
\end{aligned}
$$

The set $F^{2}$ contains only derivatives of $F^{1}$; no new equations implied by (3) are obtainable. Therefore, the chain terminates with $F^{1}$ and if there exist solutions $p_{s}{ }^{r}$ of (10), $\left|p_{s}{ }^{r}\right| \neq 0$, these solutions may be so chosen that (4) are integrable. The eliminants of the set $F^{1}$ are necessary conditions for the solution of the set $F^{1}$. These eliminants will be of the form of the equality of absolute algebraic invariants or the vanishing of tensors. All such will comprise the set $F^{1}$ and will be finite equations that must be satisfied.

We have reduced the problem to the algebraic form: The necessary and sufficient condition that two differential alternating tensors $a_{r_{1}} \ldots r_{q}$ and $\bar{a}_{r_{1}} \ldots r_{q}$ be equivalent is that the pairs of alternating tensors $a_{r_{1} \ldots r_{q}}$, $a_{r_{1} \ldots r_{q}^{s}}$ and $\bar{a}_{r_{1} \ldots r_{q}}, \bar{a}_{r_{1} \ldots r_{q}}$ be algebraically equivalent.

A complete set of tensors is a set in terms of which the equivalence problem is expressible. The tensors $a_{r_{1} \ldots r_{q}}$ and $a_{r_{1} \ldots r_{q} s}$, therefore constitute a complete set of tensors of the basic tensor.

5. On covariant differentiation. From the equations (7) defining the $a_{r_{1} \ldots r_{q} \varepsilon}$ in terms of the derivative of the $a_{r_{1} \ldots r_{q}}$, it might be supposed that the relations were reciprocal. That they are not may be seen by a count of the related quantities, the $a_{r_{1} \ldots r_{q} s}$ being antisymmetric in $q+1$ indices while the derivatives of the $a_{r_{1}} \ldots r_{q}$ are antisymmetric in but $q$ indices.

Since the number of independent components of the first derived tensor is less than the number of first partial derivatives of the basic tensor, we may infer that covariant differentiation is impossible. For, if first covariant derivatives were obtainable, the number of independent covariant derivatives would agree with the number of first derivatives and the existence of solutions $p_{s}{ }^{r}$ of the equations (10) relating the fundamental system of tensors in two coordinate systems would imply that these same $p$ 's would satisfy the transformation equations of (2) and their covariant derivatives. But this is impossible, for the latter set would be composed of a greater number of independent equations. 
6. Significance of derived tensor. The analytic significance of the vanishing of the first derived tensor may be easily demonstrated. An interpretation is suggested by the observation that constant values of the base tensor imply the vanishing of the first derived tensor.

Let the given tensor be $a_{r_{1} \ldots r_{q}}$ satisfying $a_{r_{1} \ldots . r_{q}}=0$. We may now regard the equations (2) as equations of condition, the $\bar{a}$ 's unassigned and the $p_{s}{ }^{r}$ to be determined. Choose arbitrary constant $p$ 's such that $\left|p_{s}{ }^{r}\right| \neq 0$, fixed $a$ 's at a point $P$, and determine the corresponding $\bar{a}$ 's. Define these constant values to be the $\bar{a}$ 's in the neighborhood of $P$. The $\bar{a}_{r_{1} \ldots r_{q} \varepsilon}$ will vanish identically and the second set of equations (10) is identically satisfied. Therefore the chain is completed with the single set (2), which, regarded as differential equations, are integrable. For the constants were chosen so that $\left|p_{s^{r}}\right| \neq 0$ for constant values of the $p$ 's at the arbitrary point $P$, and from continuity considerations the $p$ 's have values in the neighborhood satisfying this condition and equations (2). From the general theorem it follows that these quantities may be selected so that the equations (4) are integrable, yielding (1).

We have proved that the ranishing of the tensor $a_{r_{1}} \ldots r_{q}{ }^{s}$ is a necessary and sufficient condition that a coordinate system exist in which the $a_{r_{1} \ldots r_{q}}$ are constants. From the theorem that the second derived tensor of an alternating tensor vanishes identically we have the following interesting restatement of the present theorem: A coordinate system always exists in which a derived tensor is constant.

From the remark that the first derived tensor of a completely alternating tensor (of order $n$ ) vanishes identically we infer the corollary of the above theorem: A coordinate system exists for which a completely alternating tensor is constant.

7. Remark on generalized Green's Theorem. From this theorem the well known extension of Green's Theorem may be inferred.* The problem of stepping down the invariant integral of order $q+1$ to an integral of order $q$ requires a condition which may be expressed in the language of this paper as requiring that the given tensor $B$ determining the in tegrand of the integral of $(q+1)$ th order be a derived tensor $\dagger$ of some tensor $A$. In this case coordinates may be chosen for which the integrand is constant and the integration is immediate.

UNIVERSITY OF WASHINGTON

* Philip Franklin, Multiple integrals in n-space, Annals of Mathematics, (2), vol. 24 (1922-1923), pp. 213-226.

$\dagger$ See equations (37) of Franklin's paper. 